\title{
Serum gamma-glutamyl transferase to alanine aminotransferase ratio predict the vascular invasion and outcome in hepatitis $B$ virus related hepatocellular carcinoma
}

\section{Zhifeng Zhao}

Shanghai Jiao Tong University School of Medicine Affiliated Ninth People's Hospital

Jiayun Lin

Shanghai Jiao Tong University School of Medicine Affiliated Ninth People's Hospital

Xiaochun Ni

Shanghai Jiao Tong University School of Medicine Affiliated Ninth People's Hospital Hongjie Li

Shanghai Jiao Tong University School of Medicine Affiliated Ninth People's Hospital

\section{Lei Zheng}

Shanghai Jiao Tong University School of Medicine Affiliated Ninth People's Hospital

Chihao Zhang

Shanghai Jiao Tong University School of Medicine Affiliated Ninth People's Hospital

Xiaoliang Qi

Shanghai Jiao Tong University School of Medicine Affiliated Ninth People's Hospital

\section{Haizhong Huo}

Shanghai Jiao Tong University School of Medicine Affiliated Ninth People's Hospital

\section{Xiaolou Lou}

Shanghai Jiao Tong University School of Medicine Affiliated Ninth People's Hospital

\section{Qiang Fan}

Shanghai Jiao Tong University School of Medicine Affiliated Ninth People's Hospital

\section{Yongyang Bao}

Shanghai Jiao Tong University School of Medicine Affiliated Ninth People's Hospital

Meng Luo ( $\square$ luosh9hospital@sina.com)

Shanghai Jiao Tong University School of Medicine Affiliated Ninth People's Hospital https://orcid.org/0000-0003-3975-7476

\section{Research Article}

Keywords: gamma-glutamyl transferase, alanine aminotransferase, vascular invasion, hepatocellular carcinoma 
Posted Date: April 26th, 2021

DOl: https://doi.org/10.21203/rs.3.rs-439544/v1

License: (c) (1) This work is licensed under a Creative Commons Attribution 4.0 International License. Read Full License 


\section{Abstract}

Backgrounds: The ratio of gamma-glutamyl transferase (GGT) to alanine aminotransferase (ALT) is a predictive biomarker for hepatitis and hepatocellular carcinoma (HCC). In this study, the relationship between GGT/ALT ratio and vascular invasion was explored in hepatitis B virus (HBV)-related HCC and tumor prognosis.

Methods: Totally 558 patients were involved in this study. Univariate and multivariate logistic analysis were used to evaluate GGT/ALT as the risk factor of vascular invasion. Prognostic value of GGT/ALT was investigated by univariate and multivariate Cox analysis combined with Kaplan Meier curves. In order to reduce confounding bias, subgroup analysis and propensity score matching (PSM) were performed.

Results: Patients were divided into high and low GGT/ALT groups with an optimal cut-off value of 2.95 in predicting vascular invasion. In univariate and multivariate logistic regression, high GGT/ALT group was listed as the independent risk factors for vascular invasion $(P=0.03)$, the other risk factors included age $(P=0.001)$, a-fetoprotein (AFP) $(P=0.026)$, tumor size $(P<0.001)$, tumor capsule $(P=0.018)$, pathological differentiation $(P<0.001)$ and Barcelona Clinic Liver Cancer (BCLC) classification $(P<0.001)$. In survival analysis, high GGT/ALT ratio was associated with decreased overall survival (OS) (HR: 1.38; 95\% Cl: 1.03, 1.87; $P<0.0001)$ and disease-free survival (DFS) rates (HR: 1.32; $95 \% \mathrm{Cl}: 1.03,1.87 ; P<0.0001)$. In sensitivity analysis, comparable results were furtherly confirmed by subgroup analysis. In PSM analysis, GGT/ALT was still associated with vascular invasion independently $(\mathrm{OR}, 186 ; 95 \% \mathrm{Cl}, 1.23,3.33)$.

Conclusion: Preoperative GGT/ALT has good predictive value for vascular invasion, tumor severity and outcome in HBV-related HCC patients.

\section{Introduction}

In 2018, liver cancer was ranked as sixth most common cancer and fourth leading cause of cancer-related death in the world, with 841,000 new cases and 782,000 deaths each year[1], among which $85 \sim 90 \%$ cases belongs to hepatocellular carcinoma (HCC). Hepatitis B virus (HBV) is one of the major risk factors associated with HCC worldwide especially in China[2].

At present, the treatment of HCC mainly involves chemotherapy, surgical resection and liver transplantation[3]. However, high rates of metastasis and recurrence after operation deteriorate the prognosis severely, in this process vascular invasion accounts for the leading cause[4, 5]. Kunutsor et al.[6] demonstrated that the intrahepatic vascular invasion occupied the prevalent cause of post-operative recurrence and cancer-related death in HCC patients. Therefore, accurate and effective assessment of vascular invasion before surgery is strongly needed to guide the treatment options.

Currently, preoperative assessment of vascular invasion is mainly performed by Computed Tomography (CT), while laboratory tests are equally indispensable for adjuvant assessment. Risk factors for HCC vascular invasion include HBV infection, tumor size, multifocal localization, a-fetoprotein (AFP), y-glutamyl transferase (GGT), alanine transaminase (ALT) etc. $[4,7,8]$. As a major etiological factor, HBV infection 
changes the hepatic microenvironment, induces inflammatory response, promote the angiogenesis and vascular invasion. Several studies have confirmed the correlation between HBV infection and vascular invasion in $\mathrm{HCC}[9,10]$. Therefore, it is possible to determining vascular invasion and poor prognosis by assessing HBV severity, in which GGT and ALT have been widely investigated with considerable potential.

High GGT/ALT ratio was initially found to be prognostically associated with worse condition and treatment response in viral hepatitis[11, 12]. Further studies also indicated GGT/ALT as a positive predictor of HCC [13, 14]. However, those previous studies emphasized more on the prediction of hepatitis or HCC, while the prognostic effect of GGT/ALT on vascular invasion in HBV-related HCC is still unknown. Considering the vital role of hepatitis B in vascular invasion of HCC combining with the diagnostic value of GGT/ALT in hepatitis, the aim of our study is to confirm GGT/ALT as the risk factor for vascular invasion, cancer severity and outcomes of HBV-related HCC patients.

\section{Method}

\section{Patients}

Clinical data were collected from 558 patients with HBV infected HCC who underwent surgical resection at Zhongshan Hospital affiliated to Fudan University (Shanghai, China) between August 1, 2011 and July 31, 2017. Inclusion criteria were listed as follows: 1) HCC was diagnosed by postoperative pathological examinations; 2) history of HBV infection; 3) patient underwent hepatectomy as initial treatment in Zhongshan hospital; 4) aged over 18; 5) no distant metastasis was detected. Exclusion criteria included: 1) patients with alcoholic liver disease, hepatitis $C$ or other primary liver chronic diseases except Hepatitis $B ; 2$ ) patients with other concomitant or previous cancer; 3) patients with preoperative anti-cancer treatment such as transarterial chemoembolization (TACE), chemotherapy, radiotherapy, etc.; 4) incomplete clinical and follow-up medical records.

After the discharge, patients were followed up regularly every three months for the first three years and every six months thereafter. Serum biomarkers of tumor and hepatitis B were examined periodically, and abdominal contrast-enhanced $\mathrm{CT}$, ultrasound, hepatic arteriogram or invasive examination were performed as needed. Overall survival (OS) was defined as the interval between the date of surgery and death or the last follow-up. Disease-free survival (DFS) was defined as the time interval between the date of surgery and the date of confirmed HCC recurrence or the date of last follow-up. The last follow-up ended on July 31, 2020. The study was approved by the Ethical Committees of Zhongshan Hospital of Fudan University.

\section{Data collection}

The following clinical data of HBV infected HCC patients were collected in our study: 1) demographic data, including age and gender; 2 ) preoperative laboratory examination, including GGT, ALT, albumin, AFP and total bilirubin (TB); 3) Tumor information evaluated by imaging test, pathological examination and scoring system, including vascular invasion, tumor size, tumor multifocality, tumor capsule, pathological differentiation, cirrhosis, Barcelona Clinic Liver Cancer (BCLC) classification, Albumin-Bilirubin (ALBI) Grade; (4) Postoperative follow-up data including OS and DFS.

\section{Definition}


Laboratory examination data were manipulated by the laboratory Department of Zhongshan Hospital. The infection of HBV was defined as positive test of hepatitis $B$ virus surface antigen (HBsAg). The normal ranges of laboratory test include ALT $\leq 56 \mathrm{U} / \mathrm{L}, \mathrm{GGT} \leq 50 \mathrm{U} / \mathrm{L}$, albumin $\leq 40 \mathrm{~g} / \mathrm{L}, \mathrm{TB} \leq 20 \mu \mathrm{mol} / \mathrm{L}$, and AFP $\leq 400 \mathrm{ng} / \mathrm{mL}$, respectively. Tumor size, tumor number, tumor capsule, pathological differentiation, cirrhosis and vascular invasion were confirmed by $\mathrm{CT}$ imaging combined with postoperative pathological examination. Each specimen was reviewed independently by two liver pathologists. Microvascular invasion (MVI) is defined as microscopically confirmed tumor clusters into the vascular cavity[4]. The assessment of BCLC classification was performed as described previously[15]. Pathological differentiation was conducted using the Edmondson grading system[16]. ALBI grade was employed as a powerful tool for survival analysis in HCC patients, with specific calculation process as described previously[17].

\section{Statistical analysis}

The data analysis was performed by R (R Statistical Software, version 4.0.2). The optimal GGT/ALT cut-off value of receiver operating characteristic (ROC) curve was identified by calculating the maximum value of Youden index. Categorical variables were assessed using Pearson chi-square test or Fisher's exact test as appropriate. Logistic regression model was used for univariate and multivariate analysis. Cumulative survival rates were calculated by Kaplan-Meier and compared with the log-rank method. The Cox method was used for univariate and multivariate survival analysis. Subgroup analysis is conducted to compare the heterogeneity of prediction in subgroups. $P<0.05$ was considered statistically significant.

\section{Propensity score matching (PSM)}

To reduce bias, propensity score matching (PSM) was used. The matching process was applied by minimum distance scoring method combined with matched 1:1 match within high and low GGT/ALT group. Logistic regression models were used to calculate propensity scores, including age, sex, ALT, albumin, TB, AFP, cirrhosis, tumor size, number of tumor number, tumor capsule, pathological differentiation, BCLC classification, GGT and ALBI grade. By using Nearest-Neighbor PSM algorithm, 93 patients in high GGT/ALT group were matched 1:1 with 93 patients in low GGT/ALT group. After the matching, univariate logistic analysis was performed between high and low GGT/ALT groups in predicting the vascular invasion.

\section{Results}

\section{General clinical characteristics}

In our study, totally $558 \mathrm{HCC}$ patients with HBV infection were involved. All of the patients received tumor resection surgery and general clinical characteristics were summarized in Table 1. In those 558 patients, most patients were less than 60 years ( $75.63 \%$ vs. $24.37 \%)$, men ( $84.95 \%$ vs. $15.05 \%)$, ALT $\leq 56 \mathrm{U} / \mathrm{L}(84.41 \%$ vs. $15.59 \%)$, GGT $>50 \mathrm{U} / \mathrm{L}$ (51.25\% vs. $48.75 \%)$, albumin $>40 \mathrm{~g} / \mathrm{L}(51.97 \%$ vs. $48.03 \%), \mathrm{TB}<20 \mu \mathrm{mol} / \mathrm{L}$ ( $90.14 \%$ vs. $9.86 \%)$, AFP $<400 \mathrm{ng} / \mathrm{mL}$ (72.04\% vs. $27.96 \%)$, cirrhosis $(79.21 \%$ vs. $20.79 \%)$, tumor size $\leq 5 \mathrm{~cm}$ ( $64.52 \%$ vs. $35.48 \%$ ), solitary tumor ( $83.87 \%$ vs. $16.13 \%)$, no vascular invasion $(69.71 \%$ vs. $30.29 \%)$, tumor capsule absence (63.44\% vs. $36.56 \%$ ), and moderate/poor differentiation (67.03\% vs. $32.97 \%)$. In BCLC classification, grade A, B, C accounted for $61.65 \%, 31.54 \%$ and $6.81 \%$ separately. In ALBI grade, grade A and B accounted for $71.51 \%$ and $28.50 \%$ respectively. 


\section{Prediction value of GGT/ALT ratio for vascular invasion in HCC}

We used logistic univariate analysis and multivariate analysis to calculate the correlation between clinical information and vascular invasion. In univariate analysis, we found age (OR: 0.46, 95\% Cl: 0.29, 0.74; $P<$ 0.001), serum AFP (OR: 2.3; 95\%Cl: 1.56, 3.4; $P<0.001)$, tumor size (OR: $2.96 ; 95 \% \mathrm{Cl}: 2.04,4.31 ; P<0.001)$, tumor number (OR: 1.68; 95\% Cl: 1.05, 2.68; $P=0.032$ ), tumor capsule (OR: 1.79; 95\%Cl: 1.24, 2.59; $P=0.002$ ), pathological differentiation (OR: 3.44; 95\%Cl: 2.35, 5.04; $P<0.001$ ), BCLC B(OR: 2.95; 95\%Cl: 2.46, 3.43; $P<$ 0.001), BCLC C(OR: 3.02; 95\%Cl: 2.63, 3.72; $P<0.0001)$; ALBI B(OR: 1.95; 95\%Cl: 1.23, 2.67; $P=0.003$ ), and GGT/ALT (OR: 1.96; 95\%Cl: 1.34, 2.87; $P=0.03$ ) were associated with vascular invasion. In further multivariate logistic regression, we found that the independent risk factors for vascular invasion included age (OR: $0.44,95 \% \mathrm{Cl}: 0.27,0.74 ; P=0.001$ ), serum AFP (OR: $1.63 ; 95 \% \mathrm{Cl}: 1.06,2.51 ; P=0.026)$, tumor size (OR: $2.15 ; 95 \% \mathrm{Cl}: 1.42,3.24 ; P<0.001$ ), tumor capsule (OR: 1.64; 95\%Cl: 1.09, 2.47; $P=0.018$ ), pathological differentiation (OR: $2.90 ; 95 \% \mathrm{Cl}: 1.93,4.36 ; P<0.001)$, BCLC B(OR: 2.62; 95\%Cl: 2.21, 3.03; $P<0.001$ ), BCLC C(OR: 2.76 95\%Cl: 2.33, 3.15; $P<0.001$ ), and GGT/ALT (OR: 1.60, 95\%Cl: 1.05, 2.43; $P=0.03$ ). The results appeared that GGT/ALT was an independent risk factor for predicting vascular invasion in HCC, as shown in Table 2.

Subsequently, we plotted the ROC curve of GGT/ALT ratio in the diagnosis of vascular invasion (Fig. 1). The optimal cut-off value of GGT/ALT in the diagnosis of vascular invasion was 2.95 by calculating the Youden index of the ROC curve. Based on this cut-off value of GGT/ALT, we classified patients into low group ( $n=$ $365)$ and high group $(n=193)$.

\section{Relationship between GGT/ALT and clinicopathological parameters}

The correlations between GGT/ALT group and clinicopathological parameters were examined, which is shown in Table 1. The results indicated that the up-regulation of GGT/ALT was associated with male ( $P=$ $0.003), \mathrm{ALT}<56 \mathrm{U} / \mathrm{L}(P<0.001), \mathrm{GGT}>50 \mathrm{U} / \mathrm{L}(P<0.001)$, tumor volume $>5 \mathrm{~cm}(P<0.001)$, vascular invasion $(P<0.001)$, moderate/poor pathological differentiation $(P=0.042)$, more severe BCLC $(P<0.001)$ and ALBI grade $(P=0.007)$.

Next, by using subgroup analysis, we explored the heterogenetic prediction value of GGT/ALT in different subgroups (Table 3). The results showed that GGT/ALT had better predictive effects in subgroups of age both $\leq$ 60years (OR: 1.75; 95\% Cl: 1.14, 2.68; $P=0.011$ ) and $>60$ years (OR: $3.42 ; 95 \% \mathrm{Cl}: 1.41,8.26 ; P=$ 0.006), male (OR: 2.2; 95\%Cl: 1.47, 3.29; $P<0.001$ ), albumin $\leq 40 \mathrm{~g} / \mathrm{L}$ (OR: $2.25 ; 95 \% \mathrm{Cl}: 1.33,3.81 ; P=0.003$ ), TB $\leq 20 \mu \mathrm{mol} / \mathrm{L}$ (OR: 1.94; 95\%Cl: 1.3, 2.9; $P=0.001)$, AFP $\leq 400 \mathrm{ng} / \mathrm{mL}$ (OR: $2.42 ; 95 \% \mathrm{Cl}: 1.51,3.88 ; P<$ 0.001 ), cirrhosis (OR: $1.91 ; 95 \% \mathrm{Cl}: 1.25,2.92 ; P=0.003)$, tumor size $\leq 5 \mathrm{~cm}$ (OR: $2.18 ; 95 \% \mathrm{Cl}: 1.27,3.75 ; P=$ 0.005), solitary tumor (OR: $1.92 ; 95 \% \mathrm{Cl}: 1.26,2.93 ; P=0.003)$, tumor capsule presence (OR: $1.86 ; 95 \% \mathrm{Cl}: 1.02$, 3.4; $P=0.044$ ) or absence (OR: $2.07 ; 95 \% \mathrm{Cl}: 1.26,3.4 ; P=0.004$ ), moderate/poor pathological differentiation (OR: $2.16 ; 95 \% \mathrm{Cl}: 1.29,3.64 ; P=0.004)$, and ALBI stage A (OR: 1.93; 95\%Cl: 1.2, 3.11; $P=0.007$ ). Besides, high GGT/ALT values had a stronger positive correlation with the subgroups above.

\section{Survival analysis}

By using univariate and multivariate Cox regression analysis, we found that larger tumor size (OR: 2.27; $95 \% \mathrm{Cl}: 1.25,4.15 ; P=0.007$ ), moderate/poor differentiation (OR: 1.36; 95\%Cl: $1.01,1.83 ; P=0.040$ ), higher 
GGT/ALT (OR: 1.38; 95\%Cl: 1.03, 1.87; $P=0.033$ ), and BCLC grade C (OR: $2.75 ; 95 \% \mathrm{Cl}: 1.51,5.04 ; P=0.001$ ) were potential prognostic factors for predicting OS in HCC, as shown in Table 4. Subsequently, OS curve of GGT/ALT was drawn with a total follow-up time of 72 months. The results illustrated that the OS rate of higher GGT/ALT group was significantly lower $(P<0.0001)$ during the whole follow-up time. The 5-year OS rates is $56.07 \%$ in high group and $72.73 \%$ in low group, as shown in Fig. 2.

Cox regression analysis of DFS outlined the cirrhosis (OR: 1.65;95\%Cl: 1.17, 2.31; $P=0.004$ ), larger tumor size (OR: 1.85; 95\%Cl: 1.09, 3.15; $P=0.024$ ), higher GGT/ALT (OR: 1.32; 95\%Cl: 1.02, 1.70; $P=0.031$ ), and BCLC grade C (OR: $2.42 ; 95 \% \mathrm{Cl}: 1.44,4.07 ; P<0.001)$ as potential predictors (Table 5). The DFS curve of GGT/ALT yielded that the DFS rate of higher GGT/ALT group was significantly lower similarly with the OS rate $(P<0.0001)$, as shown in Fig. 3. The 5 -year DFS survival rates of the high and low GGT/ALT group were $43.35 \%$ and $58.70 \%$, respectively.

\section{PSM of GGT/ALT for vascular invasion prediction}

To avoid the interference of other clinical parameters, PSM was used to match the data of high and low GGT/ALT groups. After the matching, there were 93 patients equally in the low and high GGT/ALT group. In the original data, differences in distributions were characterized in several variables including sex (SD = $0.302)$, ALT ( $S D=0.727)$, Albumin ( $S D=0.184)$, tumor size $(S D=0.469)$, tumor differentiation $(S D=0.194)$, $B C L C(S D=0.424), G G T(S D=1.648)$ and $A L B I$ grade $(S D=0.251)$. After matching, there was no significant difference in the distribution between the two groups except cirrhosis (SD =0.132), as shown in Table 6 .

After matching, univariate logistic analysis was performed to evaluate the potential risk factor in the prediction of vascular invasion in HCC. The results showed that age (OR: $0.36 ; 95 \% \mathrm{Cl}: 0.15,0.87 ; P=0.015)$, AFP (OR: $2.11 ; 95 \% \mathrm{Cl}: 1.08,4.12 ; P=0.029)$, tumor size (OR: $2.68 ; 95 \% \mathrm{Cl}: 1.42,5.07 ; P=0.002)$, tumor capsule (OR: $2.05 ; 95 \% \mathrm{Cl}: 1.09,3.87 ; P=0.026$ ), pathological differentiation (OR: 4.71; 95\%Cl: 2.42, 9.17; $P<$ 0.001 ), BCLC grade B (OR: $2.61 ; 95 \% \mathrm{Cl}: 1.32,5.15 ; P=0.006)$, grade C (OR: $2.88 ; 95 \% \mathrm{Cl}: 2.18,3.42 ; P<0.001)$ and GGT/ALT (OR: $1.86 ; 95 \% \mathrm{Cl}: 1.23,3.33 ; P=0.037$ ) were still correlated with vascular invasion, as shown in Table 7. The results confirmed the GGT/ALT as a good predictive value for vascular invasion.

\section{Discussion}

Our study demonstrated the GGT/ALT ratio as an independent predictive biomarker for vascular invasion in HBV-related HCC. After grouping patients with cut-off value of 2.95, high GGT/ALT group showed positive predictive value for vascular invasion, higher tumor severity, lower DFS and OS in HCC patients. Further sensitivity analysis including subgroup analysis and PSM was performed and demonstrated GGT/ALT as an independent predictor for vascular invasion in HCC patients.

Vascular invasion is one of the major factors leading to poor prognosis of HCC, thus influences the treatment effect of resection surgery severely[18]. Therefore, timely and accurate evaluations are pivotal for guiding the therapeutic approaches and improving the survival. As a main etiology of HCC, infection of HBV has been proved as an important causative agent of vascular invasion in HCC patients. 
HBV infection leads to multiple pathophysiological alteration including DNA oxidative damage, liver cell necrosis, inflammation responses, cytokine synthesis and release, fibrosis and tumor tumorigenesis[19]. In this process, HBV X protein ( $\mathrm{HBX}$ ) has been found related with the MVI development involved in postoperative recurrence [20, 21]. Yang et al.[10] found that HBV-positive patients were more prone to develop vascular invasion in HCC. In this study, HBV was found to change the intrahepatic microenvironment through the transformer growth factor beta(TGF- $\beta$ ) - miR34a - C-C motif chemokine ligand 22 (CCL22) pathway, thereby promoting the angiogenesis and the development of HCC. In further clinical studies, Lei et al.[9] discovered that preoperative HBV DNA load larger than $104 \mathrm{IU} / \mathrm{mL}$ was an independent risk factor for vascular invasion in HBV-related HCC. Wei et al.[22] further summarized that both infection and active replication of HBV were associated with the inflammatory injuries, occurrence of vascular invasion and cancer metastasis in liver cancer. Therefore, the aim of our study is to predict the vascular invasion and outcomes of HCC via the assessment of HBV infection.

Among the examinations, GGT and ALT are adopted most broadly in hepatitis evaluation[23-27]. GGT could mediate the production of ROS, promote cell growth and proliferation[28], and is often elevated in liver diseases such as hepatitis, fibrosis and cancer[24, 29]. ALT is also known as a marker of liver dysfunction and inflammation, which was found associated with recurrence and poor survival of HBV-related HCC[30, 31]. However, the changes of different biomarkers were not equally accompanied with the fluctuation of inflammatory responses, therefore the combination of GGT/ALT exerts better predictive accuracy in hepatitis compared with using alone[32]. Ebiling et al.[11] disclosed that higher GGT/ALT ratio could predict the worse prognosis in chronic hepatitis $\mathrm{C}$. The research of Tarantino et al.[12] further supported this idea by the discoveries that a lower GGT/ALT ratio is an independent predictor of anti-virus therapy response. Consequently, GGT/ALT could reflect the hepatitis level both in primary disease and therapy responses, which made it possible in the prediction of hepatitis-induced HCC.

Yang et al.[14] found the incidence of primary hepatic carcinoma increased markedly when GGT/ALT elevated in chronic hepatitis B patients. Ju et al.[13] furtherly discovered that increased GGT/ALT ratio was closely related to tumor burden in HBV-infected HCC with Child-Pugh A class, including tumor size, vascular invasion, tumor capsule, and poor prognosis. Those studies proved the predictive potential of GGT/ALT not only in hepatitis but also in hepatic carcinoma. Combining the pathological role of HBV infection in vascular invasion thus in HCC progression, and the prediction value of GGT/ALT in both hepatitis B and HCC, we conducted this study to confirm GGT/ALT as an independent predictive factor of vascular invasion and outcomes in HBV-infected HCC.

As mentioned above, GGT/ALT have the ability to predict vascular invasion in HCC. However, different distribution of characteristics among HCC patients increased the heterology and might lead to false positive results. Therefore, sensitivity analysis including subgroup analysis and PSM was conducted. In subgroup analysis, the prediction value of GGT/ALT was examined effectively in most subgroups. The insignificant predictive value in other subgroups might be attributed to relatively small amount of cases and this could be confirmed through larger database in future. Moreover, PSM was employed to balance the distributions of variables between high and low GGT/ALT groups. After the matching, GGT/ALT was still listed as an independently risk factor of vascular invasion, which proved its prediction value of the ratio solidly. 
By now, there is no authoritative explanation for the prediction value of GGT/ALT. One possible hypothesis is proposed as follows: GGT is affected by both inflammation and tumors, while ALT is more responsive to the hepatic inflammation and liver functions relatively. Although the hepatitis in HCC patients has often been controlled before the resection, GGT and ALT might still be interfered by residual HBV infection or surgical stimulus. Therefore, the ratio of GGT/ALT could reflect the stages of primary tumor progression more precisely by minimizing the interference of inflammation. Besides, decreased ALT also indicated increment mortality rate in people aged over 60 years because of the depletion of liver function reserve, which could explain the positive correlation between GGT/ALT ratio and worse prognosis in another aspect[33].

Some scoring systems, such as BCLC classification or ALBI grade, have also exhibited good predicting values in HCC. However, those scoring system is relatively difficult to address, which make it limited in clinical use. As a powerful complement to imaging examination and scoring systems, our study provided a simple and feasible monitoring tool to assess the vascular invasion and outcome of HCC, which make it easier in the patients' management.

This study still had several limitations. Firstly, this study only involves a single center. More cases from different centers and regions are required in the future. Secondly, this study is a retrospective study, which may lead to selection bias. It may be further verified by prospective cohort studies in the future.

\section{Conclusion}

Our study proposed a relatively reliable evidence in proving the prognostic value of GGT/ALT. As a cheap and convenient biomarker, GGT/ALT is an independent predictor of vascular invasion, tumor severity and outcome in HBV-related HCC patients undergoing resection surgery.

\section{Abbreviations}

HCC: Hepatocellular carcinoma; HBV: Hepatitis B virus; CT: Computed Tomography; AFP: a-fetoprotein; GGT: Y-glutamyl transferase; ALT: Alanine transaminase; ROS: Reactive oxygen species; TACE: Transarterial chemoembolization; OS: Overall survival; DFS: Disease-free survival; TB: Total bilirubin; BCLC: Barcelona Clinic Liver Cancer; ALBI: Albumin-Bilirubin; HBsAg: Hepatitis B virus surface antigen; ROC: Receiver operating characteristic; PSM: Propensity score matching; MVI: Microvascular invasion.

\section{Declarations}

\section{Ethics approval and consent to participate}

The experimental protocol was established, according to the ethical guidelines of the Helsinki Declaration and was approved by the Fudan University affiliated Zhongshan Hospital Ethics Committee. Informed consent was waived for this non-interventional, observational, and retrospective study by the Ethics Committees, in which the patient data used were kept strictly confidential.

\section{Consent for publication}


Not Applicable

\section{Availability of data and material}

The datasets generated and analysed during the current study are not publicly available as the data are being used in next study, but are available from the corresponding author on reasonable request.

\section{Competing interests}

The authors declare that they have no competing interests.

\section{Funding}

This study is financially supported by the National Natural Science Fund of China (Project No. 81970526, 81900550 and 81770599).

The funding body had no role in the design of the study and collection, analysis, interpretation of data or preparation of the manuscript.

\section{Authors' contributions}

$\mathrm{ZZ}, \mathrm{YB}$ and $\mathrm{ML}$ was involved in the plan of program and drafted the manuscript. JL and XN participated the data collection and analysis. HL and LZ participated the data collection. CZ and XQ made substantial contributions to the manuscript and the analysis of data. $\mathrm{HH}, \mathrm{XL}, \mathrm{QF}$ and $\mathrm{ZZ}$ participated in drafting or revising the work. All authors have given final approval of the version to be published. All authors agreed to be accountable for all aspects of the manuscript.

\section{Acknowledgements}

We are thankful to Manghua Xu, Tao Qin, Jian Li and Dengyu Wang for provding excellent research condition and guidance.

\section{References}

1. Bray F, Ferlay J, Soerjomataram I, Siegel RL, Torre LA, Jemal A. Global cancer statistics 2018: GLOBOCAN estimates of incidence and mortality worldwide for 36 cancers in 185 countries. CA Cancer J Clin. 2018;68:394-424. doi:10.3322/caac.21492.

2. Thun M, Linet MS, Cerhan JR, Haiman CA, Schottenfeld D. Cancer Epidemiology and Prevention: Oxford University Press; 2017.

3. Poon D, Anderson BO, Chen L-T, Tanaka K, Lau WY, van Cutsem E, et al. Management of hepatocellular carcinoma in Asia: consensus statement from the Asian Oncology Summit 2009. Lancet Oncol. 2009;10:1111-8. doi:10.1016/S1470-2045(09)70241-4.

4. Rodríguez-Perálvarez M, Luong TV, Andreana L, Meyer T, Dhillon AP, Burroughs AK. A systematic review of microvascular invasion in hepatocellular carcinoma: diagnostic and prognostic variability. Ann Surg Oncol. 2013;20:325-39. doi:10.1245/s10434-012-2513-1. 
5. Shi J, Lai ECH, Li N, Guo W-X, Xue J, Lau WY, et al. Surgical treatment of hepatocellular carcinoma with portal vein tumor thrombus. Ann Surg Oncol. 2010;17:2073-80. doi:10.1245/s10434-010-0940-4.

6. Del Perea Pozo E, Bernal Bellido C, Sendín Matín M, Cepeda Franco C, Álamo Martínez JM, Suarez Artacho G, et al. Recurrent Hepatocellular Carcinoma After Liver Transplantation: Analysis of Risk Factors. Transplant Proc. 2016;48:2990-3. doi:10.1016/j.transproceed.2016.09.020.

7. Fujita N, Aishima S, Iguchi T, Mano Y, Taketomi A, Shirabe K, et al. Histologic classification of microscopic portal venous invasion to predict prognosis in hepatocellular carcinoma. Hum Pathol. 2011;42:1531-8. doi:10.1016/j.humpath.2010.12.016.

8. Zhou L, Rui J-A, Wang S-B, Chen S-G, Qu Q. Risk factors of microvascular invasion, portal vein tumor thrombosis and poor post-resectional survival in HBV-related hepatocellular carcinoma. Hepatogastroenterology. 2014;61:1696-703.

9. Lei Z, Li J, Wu D, Xia Y, Wang Q, Si A, et al. Nomogram for Preoperative Estimation of Microvascular Invasion Risk in Hepatitis B Virus-Related Hepatocellular Carcinoma Within the Milan Criteria. JAMA Surg. 2016;151:356-63. doi:10.1001/jamasurg.2015.4257.

10. Yang P, Li Q-J, Feng Y, Zhang Y, Markowitz GJ, Ning S, et al. TGF- $\beta$-miR-34a-CCL22 signaling-induced Treg cell recruitment promotes venous metastases of HBV-positive hepatocellular carcinoma. Cancer Cell. 2012;22:291-303. doi:10.1016/j.ccr.2012.07.023.

11. Ebeling F. Factors predicting interferon treatment response in patients with chronic hepatitis C: late viral clearance does not preclude a sustained response. Am J Gastroenterol. 2001;96:1237-42. doi:10.1016/S0002-9270(00)02424-2.

12. Tarantino G, Sorrentino P, Conca P, Perrella A, Ragucci P, Perrella O. Low daily dosage of interferon for 1 year after HCV-related end-therapy response. A randomized-controlled study. Liver Int. 2003;23:413-9. doi:10.1111/j.1478-3231.2003.00871.x.

13. Ju M-J, Qiu S-J, Fan J, Zhou J, Gao Q, Cai M-Y, et al. Preoperative serum gamma-glutamyl transferase to alanine aminotransferase ratio is a convenient prognostic marker for Child-Pugh A hepatocellular carcinoma after operation. J Gastroenterol. 2009;44:635-42. doi:10.1007/s00535-009-0050-x.

14. Yang J-G, He X-F, Huang B, Zhang H-A, He Y-K. Rule of changes in serum GGT levels and GGT/ALT and AST/ALT ratios in primary hepatic carcinoma patients with different AFP levels. Cancer Biomark. 2018;21:743-6. doi:10.3233/CBM-170088.

15. Llovet JM, Brú C, Bruix J. Prognosis of hepatocellular carcinoma: the BCLC staging classification. Semin Liver Dis. 1999;19:329-38. doi:10.1055/s-2007-1007122.

16. EDMONDSON HA, STEINER PE. Primary carcinoma of the liver: a study of 100 cases among 48,900 necropsies. Cancer. 1954;7:462-503. doi:10.1002/1097-0142(195405)7:3<462::aidcncr2820070308>3.0.c0;2-e.

17. Johnson PJ, Berhane S, Kagebayashi C, Satomura S, Teng M, Reeves HL, et al. Assessment of liver function in patients with hepatocellular carcinoma: a new evidence-based approach-the ALBI grade. J Clin Oncol. 2015;33:550-8. doi:10.1200/JC0.2014.57.9151.

18. Li S-H, Guo Z-X, Xiao C-Z, Wei W, Shi M, Chen Z-Y, et al. Risk factors for early and late intrahepatic recurrence in patients with single hepatocellular carcinoma without macrovascular invasion after 
curative resection. Asian Pac J Cancer Prev. 2013;14:4759-63. doi:10.7314/apjcp.2013.14.8.4759.

19. Brechot C, Kremsdorf D, Soussan P, Pineau P, Dejean A, Paterlini-Brechot P, Tiollais P. Hepatitis B virus (HBV)-related hepatocellular carcinoma (HCC): molecular mechanisms and novel paradigms. Pathol Biol (Paris). 2010;58:278-87. doi:10.1016/j.patbio.2010.05.001.

20. Chen L, Zhang Q, Chang W, Du Y, Zhang H, Cao G. Viral and host inflammation-related factors that can predict the prognosis of hepatocellular carcinoma. Eur J Cancer. 2012;48:1977-87.

doi:10.1016/j.ejca.2012.01.015.

21. Xu J, Liu H, Chen L, Wang S, Zhou L, Yun X, et al. Hepatitis B virus X protein confers resistance of hepatoma cells to anoikis by up-regulating and activating p21-activated kinase 1. Gastroenterology. 2012;143:199-212.e4. doi:10.1053/j.gastro.2012.03.053.

22. Wei X, Li N, Li S, Shi J, Guo W, Zheng Y, Cheng S. Hepatitis B virus infection and active replication promote the formation of vascular invasion in hepatocellular carcinoma. BMC Cancer. 2017;17:304. doi:10.1186/s12885-017-3293-6.

23. Wu S-J, Lin Y-X, Ye H, Xiong X-Z, Li F-Y, Cheng N-S. Prognostic value of alkaline phosphatase, gammaglutamyl transpeptidase and lactate dehydrogenase in hepatocellular carcinoma patients treated with liver resection. Int J Surg. 2016;36:143-51. doi:10.1016/j.ijsu.2016.10.033.

24. Ma H, Zhang L, Tang B, Wang Y, Chen R, Zhang B, et al. Y-Glutamyltranspeptidase is a prognostic marker of survival and recurrence in radiofrequency-ablation treatment of hepatocellular carcinoma. Ann Surg Oncol. 2014;21:3084-9. doi:10.1245/s10434-014-3724-4.

25. Zhao W-C, Fan L-F, Yang N, Zhang H-B, Chen B-D, Yang G-S. Preoperative predictors of microvascular invasion in multinodular hepatocellular carcinoma. Eur J Surg Oncol. 2013;39:858-64. doi:10.1016/j.ejso.2013.04.003.

26. Wang K, Li L, Wu Y, Yang Y, Chen J, Zhang D, et al. Increased serum gamma-glutamyltransferase levels are associated with ventricular instability in type 2 diabetes. Endocrine. 2016;52:63-72. doi:10.1007/s12020-015-0760-1.

27. Kim WR, Flamm SL, Di Bisceglie AM, Bodenheimer HC. Serum activity of alanine aminotransferase (ALT) as an indicator of health and disease. Hepatology. 2008;47:1363-70. doi:10.1002/hep.22109.

28. Kunutsor SK, Apekey TA, van Hemelrijck M, Calori G, Perseghin G. Gamma glutamyltransferase, alanine aminotransferase and risk of cancer: systematic review and meta-analysis. Int $\mathrm{J}$ Cancer. 2015;136:1162-70. doi:10.1002/ijc.29084.

29. Silva ISS, Ferraz MLCG, Perez RM, Lanzoni VP, Figueiredo VM, Silva AEB. Role of gamma-glutamyl transferase activity in patients with chronic hepatitis $C$ virus infection. J Gastroenterol Hepatol. 2004;19:314-8. doi:10.1111/j.1440-1746.2003.03256.x.

30. Tarao K, Takemiya S, Tamai S, Sugimasa Y, Ohkawa S, Akaike M, et al. Relationship between the recurrence of hepatocellular carcinoma (HCC) and serum alanine aminotransferase levels in hepatectomized patients with hepatitis C virus-associated cirrhosis and HCC. Cancer. 1997;79:688-94. doi:10.1002/(SICI)1097-0142(19970215)79:4<688::AID-CNCR5>3.0.C0;2-A.

31. Cheung Y-S, Chan HL, Wong J, Lee K-F, Poon TC, Wong N, Lai PB. Elevated Perioperative Transaminase Level Predicts Intrahepatic Recurrence in Hepatitis B-related Hepatocellular Carcinoma After Curative 
Hepatectomy. Asian J Surg. 2008;31:41-9. doi:10.1016/S1015-9584(08)60056-1.

32. Lippi G, Montagnana M, Salvagno GL, Guidi GC. Influence of stable, long-term treatment with phenobarbital on the activity of serum alanine aminotransferase and gamma-glutamyltransferase. $\mathrm{Br} \mathrm{J}$ Biomed Sci. 2008;65:132-5. doi:10.1080/09674845.2008.11732816.

33. Oh C-M, Won Y-J, Cho H, Lee J-K, Park BY, Jun JK, et al. Alanine aminotransferase and gamma-glutamyl transferase have different dose-response relationships with risk of mortality by age. Liver Int. 2016;36:126-35. doi:10.1111/liv.12879.

\section{Tables}

Table 1 Distribution of clinical variables among total, high and low GGT/ALT groups 


\begin{tabular}{|c|c|c|c|c|}
\hline Variables & Total $(n=558)$ & low $(n=365)$ & high $(n=193)$ & $P$ \\
\hline Age, n (\%) & & & & 1 \\
\hline$\leq 60$ & $422(75.63)$ & $291(75.58)$ & $131(75.72)$ & \\
\hline$>60$ & $136(24.37)$ & $94(24.42)$ & $42(24.28)$ & \\
\hline Sex, n (\%) & & & & 0.003 \\
\hline female & $84(15.05)$ & $70(18.18)$ & $14(8.09)$ & \\
\hline male & $474(84.95)$ & $315(81.82)$ & $159(91.91)$ & \\
\hline $\mathrm{ALT}, \mathrm{n}(\%)$ & & & & $<0.001$ \\
\hline$\leq 56$ & $471(84.41)$ & $299(77.66)$ & $172(99.42)$ & \\
\hline$>56$ & $87(15.59)$ & $86(22.34)$ & $1(0.58)$ & \\
\hline Albumin, n (\%) & 0.057 & & & \\
\hline$\leq 40$ & $268(48.03)$ & $211(54.81)$ & $79(45.66)$ & \\
\hline$>40$ & $290(51.97)$ & $174(45.19)$ & $94(54.34)$ & \\
\hline TB, n (\%) & 0.657 & & & \\
\hline$\leq 20$ & $503(90.14)$ & $349(90.65)$ & $154(89.02)$ & \\
\hline$>20$ & $55(9.86)$ & $36(9.35)$ & $19(10.98)$ & \\
\hline AFP, n (\%) & 0.399 & & & \\
\hline$\leq 400$ & $402(72.04)$ & $282(73.25)$ & $120(69.36)$ & \\
\hline$>400$ & $156(27.96)$ & $103(26.75)$ & $53(30.64)$ & \\
\hline Cirrhosis, n (\%) & 0.904 & & & \\
\hline no & 116 (20.79) & $79(20.52)$ & 37 (21.39) & \\
\hline yes & $442(79.21)$ & $306(79.48)$ & $136(78.61)$ & \\
\hline Size, n (\%) & $<0.001$ & & & \\
\hline$\leq 5$ & $360(64.52)$ & $272(70.75)$ & $88(50.87)$ & \\
\hline$>5$ & $198(35.48)$ & $113(29.35)$ & $85(49.13)$ & \\
\hline Number, n (\%) & 0.518 & & & \\
\hline solitary & 468 (83.87) & 326 (84.68) & $142(82.08)$ & \\
\hline multiple & $90(16.13)$ & $59(15.32)$ & $31(17.92)$ & \\
\hline Vascular invasion, $\mathrm{n}(\%)$ & $<0.001$ & & & \\
\hline no & $389(69.71)$ & $286(74.29)$ & $103(59.54)$ & \\
\hline
\end{tabular}




\begin{tabular}{llll} 
yes & $169(30.29)$ & $99(25.71)$ & $70(40.46)$ \\
Tumor capsule, n (\%) & 0.962 & & \\
absence & $354(63.44)$ & $245(63.64)$ & $109(63.01)$ \\
presence & $204(36.56)$ & $140(36.37)$ & $64(36.99)$ \\
Differential, n (\%) & 0.042 & & \\
good & $184(32.97)$ & $269(69.87)$ & $105(60.69)$ \\
moderate/poor & $374(67.03)$ & $116(30.13)$ & $68(39.31)$ \\
BCLC, n (\%) & $<0.001$ & & \\
A & $344(61.65)$ & $260(67.53)$ & $84(48.55)$ \\
B & $176(31.54)$ & $108(28.05)$ & $68(39.30)$ \\
C & $38(6.81)$ & $17(4.42)$ & $21(12.14)$ \\
GGT, n (\%) & $<0.001$ & & $11(6.36)$ \\
550 & $272(48.75)$ & $261(67.79)$ & $162(93.64)$ \\
$>50$ & $286(51.25)$ & $124(32.21)$ & \\
ALBI Grade, n (\%) & 0.007 & & $63(36.42)$ \\
A & $399(71.51)$ & $289(75.06)$ & $110(63.58)$ \\
B & $159(28.50)$ & $96(24.90)$ & \\
\hline
\end{tabular}

ALT, Alanine aminotransferase; TB, Total Bilirubin; AFP, $\alpha$-fetoprotein; BCLC, Barcelona Clinic Liver Cancer staging; GGT, $\gamma$-Glutamyl Transpeptidase; ALBI grade, Albumin-Bilirubin Grade.

Table 2 Univariate and multivariate logistic analysis of prognostic factors associated with vascular invasion 


\begin{tabular}{lllll}
\hline Risks & Univariate analysis & \multicolumn{3}{l}{ Multivariate analysis } \\
& OR $(95 \% \mathrm{CI})$ & $P$ & OR $(95 \% \mathrm{CI})$ & $P$ \\
\hline
\end{tabular}

Age, n (\%)

$\begin{array}{ll}\leq 60 & \text { reference } \\ >60 & 0.46(0.29,0.74)\end{array}$

$<0.001$

reference

$\begin{array}{lll}<0.001 & 0.44(0.27,0.74) & 0.001\end{array}$

Sex, n (\%)

female reference

male

$$
1.36(0.8,2.32) \quad 0.245
$$

Albumin, n

(\%)

$\begin{array}{ll}\leq 40 & \text { reference } \\ >40 & \end{array}$

$$
1.26(0.88,1.81) \quad 0.208
$$

TB, $\mathbf{n}(\%)$

$$
\begin{array}{lll}
>20 & \text { reference } & \\
\leq 20 & 0.97(0.53,1.77) & 0.916
\end{array}
$$

\section{AFP, $\mathbf{n}(\%)$}

$\leq 400$

reference

reference

$>400$

$$
2.3(1.56,3.4)
$$

$<0.001$

$1.63(1.06,2.51)$

0.026

Cirrhosis, n

(\%)

no

reference 
yes

$1.24(0.79,1.97)$

0.344

\section{Size, n (\%)}

$\leq 5$

reference

$>5$

$$
<0.001
$$

reference

$$
1.44(0.85,2.41) \quad 0.175
$$

\section{Tumor}

capsule, n (\%)

absence reference

reference

presence

$$
1.79(1.24,2.59)
$$

0.002

$1.64(1.09,2.47)$

0.018

\section{Differential, $\mathbf{n}$}

(\%)

good

reference

reference

moderate/poor

$3.44(2.35,5.04)$

$<0.001$

$2.90(1.93,4.36)$ $<0.001$

BCLC, n (\%)
A
reference

B

$$
2.95(2.46,3.43)
$$

$<0.001$

C

$<0.001$ 


\section{ALBI Grade, $\mathbf{n}$}

(\%)

A

B

reference

$1.95(1.23,2.67)$

0.003

reference

$1.44(0.93,2.21)$

0.1

\section{GGT/ALT}

$\leq 2.95$

reference

reference

$>2.95$

$<0.001$

$1.60(1.05,2.43)$

0.030

TB, Total Bilirubin; AFP, $\alpha$-fetoprotein; BCLC, Barcelona Clinic Liver Cancer staging; ALBI grade, Albumin-Bilirubin Grade; GGT, $\gamma$-Glutamyl Transpeptidase; ALT, Alanine aminotransferase.

Table 3 Subgroup analysis for low and high GGT/ALT groups 


\begin{tabular}{|c|c|c|c|c|c|c|}
\hline $\begin{array}{l}\text { Subgroup } \\
\text { Age }\end{array}$ & \multicolumn{3}{|c|}{ Number of patients Low High } & \multicolumn{2}{|r|}{ OR $(95 \% \mathrm{Cl})$} & $\mathrm{p}$ value \\
\hline$\leq 60$ & 422 & 291 & 131 & $\mapsto$ & $1.75(1.14-2.68)$ & 0.011 \\
\hline$>60$ & 136 & 94 & 42 & $\longrightarrow$ & $3.42(1.41-8.26)$ & 0.006 \\
\hline \multicolumn{7}{|l|}{ Sex } \\
\hline female & 84 & 70 & 14 & $\longrightarrow$ & $0.45(0.09-2.19)$ & 0.287 \\
\hline male & 474 & 315 & 159 & $\mapsto$ & $2.2(1.47-3.29)$ & $<0.001$ \\
\hline \multicolumn{7}{|l|}{ Albumin } \\
\hline$\leq 40$ & 268 & 174 & 94 & $\longmapsto$ & $2.25(1.33-3.81)$ & 0.003 \\
\hline$>40$ & 290 & 211 & 79 & $\mapsto$ & $1.64(0.94-2.85)$ & 0.085 \\
\hline \multicolumn{7}{|l|}{ TB } \\
\hline$\leq 20$ & 503 & 349 & 154 & $\longmapsto$ & $1.94(1.3-2.9)$ & 0.001 \\
\hline$>20$ & 55 & 36 & 19 & 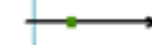 & - $2.18(0.67-7.12)$ & 0.196 \\
\hline \multicolumn{7}{|l|}{ AFP } \\
\hline$\leq 400$ & 402 & 282 & 120 & $\longmapsto$ & $2.42(1.51-3.88)$ & $<0.001$ \\
\hline$>400$ & 156 & 103 & 53 & $\mapsto$ & $1.25(0.64-2.43)$ & 0.518 \\
\hline \multicolumn{7}{|l|}{ Cirrhosis } \\
\hline no & 116 & 79 & 37 & & - $2.22(0.95-5.21)$ & 0.069 \\
\hline yes & 442 & 306 & 136 & 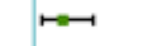 & $1.91(1.25-2.92)$ & 0.003 \\
\hline \multicolumn{7}{|l|}{ Size } \\
\hline$\leq 5$ & 360 & 272 & 88 & $\longmapsto$ & $2.18(1.27-3.75)$ & 0.005 \\
\hline$>5$ & 198 & 113 & 85 & $\mapsto$ & $1.22(0.69-2.14)$ & 0.497 \\
\hline \multicolumn{7}{|l|}{ Number } \\
\hline solitary & 468 & 326 & 142 & $\mapsto$ & $1.92(1.26-2.93)$ & 0.003 \\
\hline multiple & 90 & 59 & 31 & $\longrightarrow$ & $2.08(0.86-5.05)$ & 0.104 \\
\hline \multicolumn{7}{|c|}{ Tumor capsule } \\
\hline absence & 354 & 245 & 109 & $\longmapsto$ & $2.07(1.26-3.4)$ & 0.004 \\
\hline presence & 204 & 140 & 64 & $\longmapsto$ & $1.86(1.02-3.4)$ & 0.044 \\
\hline \multicolumn{7}{|l|}{ Differential } \\
\hline good & 184 & 116 & 68 & $\mapsto$ & $1.47(0.81-2.68)$ & 0.209 \\
\hline moderate/poor & 374 & 269 & 105 & $\longmapsto$ & $2.16(1.29-3.64)$ & 0.004 \\
\hline \multicolumn{7}{|l|}{ BCLC } \\
\hline A & 344 & 260 & 84 & $\longmapsto$ & $1.73(0.96-3.13)$ & 0.076 \\
\hline B & 176 & 108 & 68 & $\mapsto$ & $1.22(0.65-2.25)$ & 0.537 \\
\hline C & 38 & 17 & 21 & 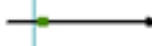 & $-1.25(0.07-21.58)$ & 0.878 \\
\hline \multicolumn{7}{|l|}{ ALBI Grade } \\
\hline A & 399 & 289 & 110 & $\longmapsto$ & $1.93(1.2-3.11)$ & 0.007 \\
\hline B & 159 & 96 & 63 & 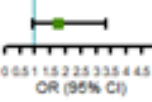 & $1.74(0.91-3.32)$ & 0.096 \\
\hline
\end{tabular}

TB, Total Bilirubin; AFP, $\alpha$-fetoprotein; BCLC, Barcelona Clinic Liver Cancer staging; ALBI grade, Albumin-Bilirubin Grade.

Table 4 Multivariate Cox regression analysis of overall survival 


\begin{tabular}{|c|c|c|c|c|c|}
\hline Variable & & $\mathbf{N}$ & Hazard ratio & & p \\
\hline \multirow[t]{2}{*}{ AFP } & $\leq 400$ & 402 & 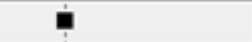 & Reference & \\
\hline & $>400$ & 156 & $=$ & $1.10(0.80,1.51)$ & 0.547 \\
\hline \multirow[t]{2}{*}{ Cirrhosis } & no & 116 & - & Reference & \\
\hline & yes & 442 & $=$ & $1.43(0.95,2.15)$ & 0.091 \\
\hline \multirow[t]{2}{*}{ Size } & $\leq 5$ & 360 & 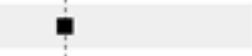 & Reference & \\
\hline & $>5$ & 198 & $\square$ & $2.27(1.25,4.15)$ & 0.007 \\
\hline \multirow[t]{2}{*}{ Number } & solitary & 468 & i & Reference & \\
\hline & multiple & 90 & $=$ & $1.34(0.95,1.91)$ & 0.098 \\
\hline \multirow{2}{*}{ Tumor capsule } & absence & 354 & 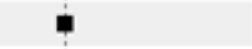 & Reference & \\
\hline & presence & 204 & - & $1.06(0.78,1.44)$ & 0.693 \\
\hline \multirow[t]{2}{*}{ Differention } & good & 374 & 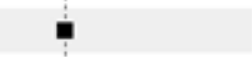 & Reference & \\
\hline & moderate/poor & 184 & - & $1.36(1.01,1.83)$ & 0.040 \\
\hline \multirow[t]{2}{*}{ GGT/ALT } & low & 385 & 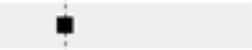 & Reference & \\
\hline & high & 173 & - & $1.38(1.03,1.87)$ & 0.033 \\
\hline \multirow[t]{2}{*}{ ALBI_Grade } & $A$ & 399 & 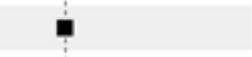 & Reference & \\
\hline & B & 159 & - & $1.15(0.85,1.56)$ & 0.375 \\
\hline \multirow[t]{3}{*}{ BCLC } & A & 344 & 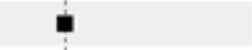 & Reference & \\
\hline & $B$ & 176 & $\longrightarrow$ & $1.05(0.56,1.97)$ & 0.888 \\
\hline & C & 38 & 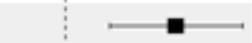 & $2.75(1.51,5.04)$ & 0.001 \\
\hline
\end{tabular}

AFP, $\alpha$-fetoprotein; GGT, $\gamma$-Glutamyl Transpeptidase; ALT, Alanine aminotransferase; ALBI grade, Albumin-Bilirubin Grade; BCLC, Barcelona Clinic Liver Cancer staging.

Table 5 Multivariate Cox regression analysis of disease-free survival

\begin{tabular}{|c|c|c|c|c|c|}
\hline Variable & & $\mathrm{N}$ & Hazard ratio & & $\mathrm{p}$ \\
\hline \multirow[t]{2}{*}{ Cirrhosis } & no & 116 & $\dot{1}$ & Reference & \\
\hline & yes & 442 & 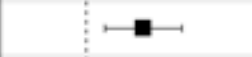 & $1.65(1.17,2.31)$ & 0.004 \\
\hline \multirow[t]{2}{*}{ Size } & $\leq 5$ & 360 & 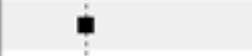 & Reference & \\
\hline & $>5$ & 198 & $\square$ & $1.85(1.09,3.15)$ & 0.024 \\
\hline \multirow[t]{2}{*}{ Number } & solitary & 468 & 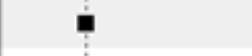 & Reference & \\
\hline & multiple & 90 & $=$ & $1.29(0.95,1.75)$ & 0.097 \\
\hline \multirow[t]{2}{*}{ GGTIALT } & low & 385 & 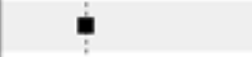 & Reference & \\
\hline & high & 173 & - & $1.32(1.02,1.70)$ & 0.031 \\
\hline \multirow[t]{2}{*}{ Differention } & good & 374 & 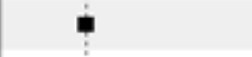 & Reference & \\
\hline & moderate/poor & 184 & $=$ & $1.26(0.99,1.61)$ & 0.064 \\
\hline \multirow[t]{2}{*}{ Tumor capsule } & absence & 354 & 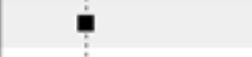 & Reference & \\
\hline & presence & 204 & - & $1.01(0.79,1.30)$ & 0.925 \\
\hline \multirow[t]{2}{*}{ ALBI_Grade } & A & 399 & 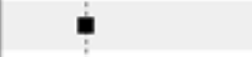 & Reference & \\
\hline & B & 159 & 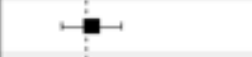 & $1.05(0.81,1.36)$ & 0.717 \\
\hline \multirow[t]{3}{*}{ BCLC } & A & 344 & $\div$ & Reference & \\
\hline & B & 176 & $\longrightarrow$ & $0.90(0.51,1.57)$ & 0.707 \\
\hline & C & 38 & $\square$ & $2.42(1.44,4.07)$ & $<0.001$ \\
\hline
\end{tabular}

GGT, $\gamma$-Glutamyl Transpeptidase; ALT, Alanine aminotransferase; ALBI grade, Albumin-Bilirubin Grade; BCLC, Barcelona Clinic Liver Cancer staging. 
Table 6 Absolute standardized differences before and after propensity score matching 


\begin{tabular}{|c|c|c|c|c|c|c|}
\hline \multirow[b]{2}{*}{ Variables } & \multicolumn{2}{|c|}{ Before matching } & \multirow[t]{2}{*}{ SD } & \multicolumn{2}{|c|}{ After matching } & \multirow[t]{2}{*}{ SD } \\
\hline & $\begin{array}{l}\text { low }(n= \\
365)\end{array}$ & $\begin{array}{l}\text { high }(n= \\
193)\end{array}$ & & $\begin{array}{l}\text { low }(n= \\
93)\end{array}$ & $\begin{array}{l}\text { high }(n= \\
93)\end{array}$ & \\
\hline Age, n (\%) & & & 0.003 & & & 0.077 \\
\hline$\leq 60$ & $291(75.58)$ & $131(75.72)$ & & $73(78.49)$ & $70(75.27)$ & \\
\hline$>60$ & $94(24.42)$ & $42(24.28)$ & & $20(21.51)$ & $23(24.73)$ & \\
\hline Sex, n (\%) & & & 0.302 & & & 0.032 \\
\hline female & $70(18.18)$ & $14(8.09)$ & & $12(12.90)$ & $13(13.98)$ & \\
\hline male & 315 (81.82) & $159(91.91)$ & & $81(87.10)$ & $80(86.02)$ & \\
\hline $\mathrm{ALT}, \mathrm{n}(\%)$ & & & 0.727 & & & $<0.001$ \\
\hline$\leq 56$ & $299(77.66)$ & $172(99.42)$ & & $92(98.92)$ & $92(98.92)$ & \\
\hline$>56$ & $86(22.34)$ & $1(0.58)$ & & $1(1.08)$ & $1(1.08)$ & \\
\hline Albumin, n (\%) & & & 0.184 & & & 0.086 \\
\hline$\leq 40$ & $211(54.81)$ & $79(45.66)$ & & $49(52.69)$ & $45(48.39)$ & \\
\hline$>40$ & $174(45.19)$ & $94(54.34)$ & & $44(47.31)$ & $48(51.61)$ & \\
\hline $\mathrm{TB}, \mathbf{n}(\%)$ & & & 0.054 & & & 0.077 \\
\hline$\leq 20$ & 349 (90.65) & $154(89.02)$ & & $86(92.47)$ & $84(90.32)$ & \\
\hline$>20$ & $36(9.35)$ & $19(10.98)$ & & $7(7.53)$ & $9(9.68)$ & \\
\hline AFP, n (\%) & & & 0.086 & & & 0.023 \\
\hline$\leq 400$ & $282(73.25)$ & $120(69.36)$ & & $65(69.89)$ & $64(68.82)$ & \\
\hline$>400$ & $103(26.75)$ & $53(30.64)$ & & $28(30.11)$ & $29(31.19)$ & \\
\hline Cirrhosis, n (\%) & & & 0.021 & & & 0.132 \\
\hline no & $79(20.52)$ & $37(21.39)$ & & $17(18.28)$ & $22(23.66)$ & \\
\hline yes & 306 (79.48) & $136(78.61)$ & & $76(81.72)$ & $71(76.34)$ & \\
\hline Size, n (\%) & & & 0.469 & & & 0.088 \\
\hline$\leq 5$ & $272(70.75)$ & $88(50.87)$ & & $61(56.99)$ & $49(52.69)$ & \\
\hline$>5$ & $113(29.35)$ & $85(49.13)$ & & $40(43.01)$ & $44(47.31)$ & \\
\hline Number, n (\%) & & & 0.07 & & & $<0.001$ \\
\hline solitary & $326(84.68)$ & $142(82.08)$ & & $77(82.80)$ & $77(82.80)$ & \\
\hline multiple & $59(15.32)$ & $31(17.92)$ & & $16(17.20)$ & $16(17.20)$ & \\
\hline
\end{tabular}


Tumor capsule, $\mathbf{n}$

0.013

0.091

(\%)

\begin{tabular}{|c|c|c|c|c|c|c|}
\hline absence & $245(63.64)$ & 109 (63.01) & & $64(68.82)$ & $60(64.52)$ & \\
\hline presence & $140(36.37)$ & $64(36.99)$ & & $29(31.18)$ & $33(35.48)$ & \\
\hline Differential, n (\%) & & & 0.194 & & & 0.067 \\
\hline good & $269(69.87)$ & $105(60.69)$ & & $32(34.41)$ & $35(37.63)$ & \\
\hline moderate/poor & $116(30.13)$ & $68(39.31)$ & & $61(65.59)$ & $58(62.37)$ & \\
\hline BCLC, n (\%) & & & 0.424 & & & 0.092 \\
\hline A & $260(67.53)$ & $84(48.55)$ & & $51(54.84)$ & $51(54.84)$ & \\
\hline B & $108(28.05)$ & $68(39.3)$ & & $37(39.78)$ & $35(37.63)$ & \\
\hline $\mathrm{C}$ & $17(4.42)$ & $21(12.14)$ & & $5(5.38)$ & $7(7.53)$ & \\
\hline GGT, n (\%) & & & 1.648 & & & $<0.001$ \\
\hline$\leq 50$ & $261(67.79)$ & $11(6.36)$ & & $11(11.83)$ & $11(11.82)$ & \\
\hline$>50$ & $124(32.21)$ & $162(93.64)$ & & $82(88.17)$ & $82(88.17)$ & \\
\hline ALBI Grade, n (\%) & & & 0.251 & & & 0.024 \\
\hline A & $289(75.06)$ & $110(63.58)$ & & $65(69.89)$ & $66(70.97)$ & \\
\hline B & $96(24.9)$ & $63(36.42)$ & & $28(30.11)$ & $27(29.03)$ & \\
\hline
\end{tabular}

ALT, Alanine aminotransferase; TB, Total Bilirubin; AFP, $\alpha$-fetoprotein; BCLC, Barcelona Clinic Liver Cancer staging; GGT, $\gamma$-Glutamyl Transpeptidase; ALBI grade, Albumin-Bilirubin Grade.

Table 7 Univariate logistic regression associated with vascular invasion after PSM 


\begin{tabular}{|c|c|c|}
\hline \multirow[t]{2}{*}{ Risks } & \multicolumn{2}{|c|}{ Univariate analysis } \\
\hline & OR $(95 \% \mathrm{CI})$ & $P$ \\
\hline \multicolumn{3}{|l|}{ Age, n (\%) } \\
\hline$\leq 60$ & reference & \\
\hline$>60$ & $0.36(0.15,0.87)$ & 0.015 \\
\hline \multicolumn{3}{|l|}{ Sex, n (\%) } \\
\hline female & reference & \\
\hline male & $1.58(0.6,4.2)$ & 0.342 \\
\hline \multicolumn{3}{|c|}{ Albumin, n (\%) } \\
\hline$\leq 40$ & reference & \\
\hline$>40$ & $1.37(0.74,2.56)$ & 0.315 \\
\hline \multicolumn{3}{|l|}{ TB, n (\%) } \\
\hline$\leq 20$ & reference & \\
\hline$>20$ & $1.6(0.5,5.12)$ & 0.417 \\
\hline \multicolumn{3}{|l|}{$\mathrm{AFP}, \mathrm{n}(\%)$} \\
\hline$\leq 400$ & reference & \\
\hline$>400$ & $2.11(1.08,4.12)$ & 0.029 \\
\hline \multicolumn{3}{|c|}{ Cirrhosis, n (\%) } \\
\hline no & reference & \\
\hline yes & $0.93(0.42,2.08)$ & 0.864 \\
\hline \multicolumn{3}{|l|}{ Size, $n(\%)$} \\
\hline$\leq 5$ & reference & \\
\hline$>5$ & $2.68(1.42,5.07)$ & 0.002 \\
\hline \multicolumn{3}{|c|}{ Number, $\mathbf{n}(\%)$} \\
\hline solitary & reference & \\
\hline multiple & $1.24(0.6,2.55)$ & 0.567 \\
\hline \multicolumn{3}{|c|}{ Tumor capsule, n (\%) } \\
\hline absence & reference & \\
\hline presence & $2.05(1.09,3.87)$ & 0.026 \\
\hline \multicolumn{3}{|c|}{ Differential, n (\%) } \\
\hline good & reference & \\
\hline & e $24 / 28$ & \\
\hline
\end{tabular}


moderate/poor

BCLC, n (\%)
A
reference
B
C
$2.61(1.32,5.15) \quad 0.006$
$2.88(2.18,3.42)<0.001$

ALBI Grade, n (\%)
A
reference
B
$1.27(0.65,2.46) \quad 0.485$

\section{GGT/ALT}

$$
\begin{aligned}
& \leq 2.95 \\
& \text { reference } \\
& \begin{array}{lll}
>2.95 & 1.86(1.23,3.33) & 0.037
\end{array}
\end{aligned}
$$

TB, Total Bilirubin; AFP, $\alpha$-fetoprotein; BCLC, Barcelona Clinic Liver Cancer staging; ALBI grade, Albumin-Bilirubin Grade; GGT, y-Glutamyl Transpeptidase; ALT, Alanine aminotransferase.

\section{Figures}




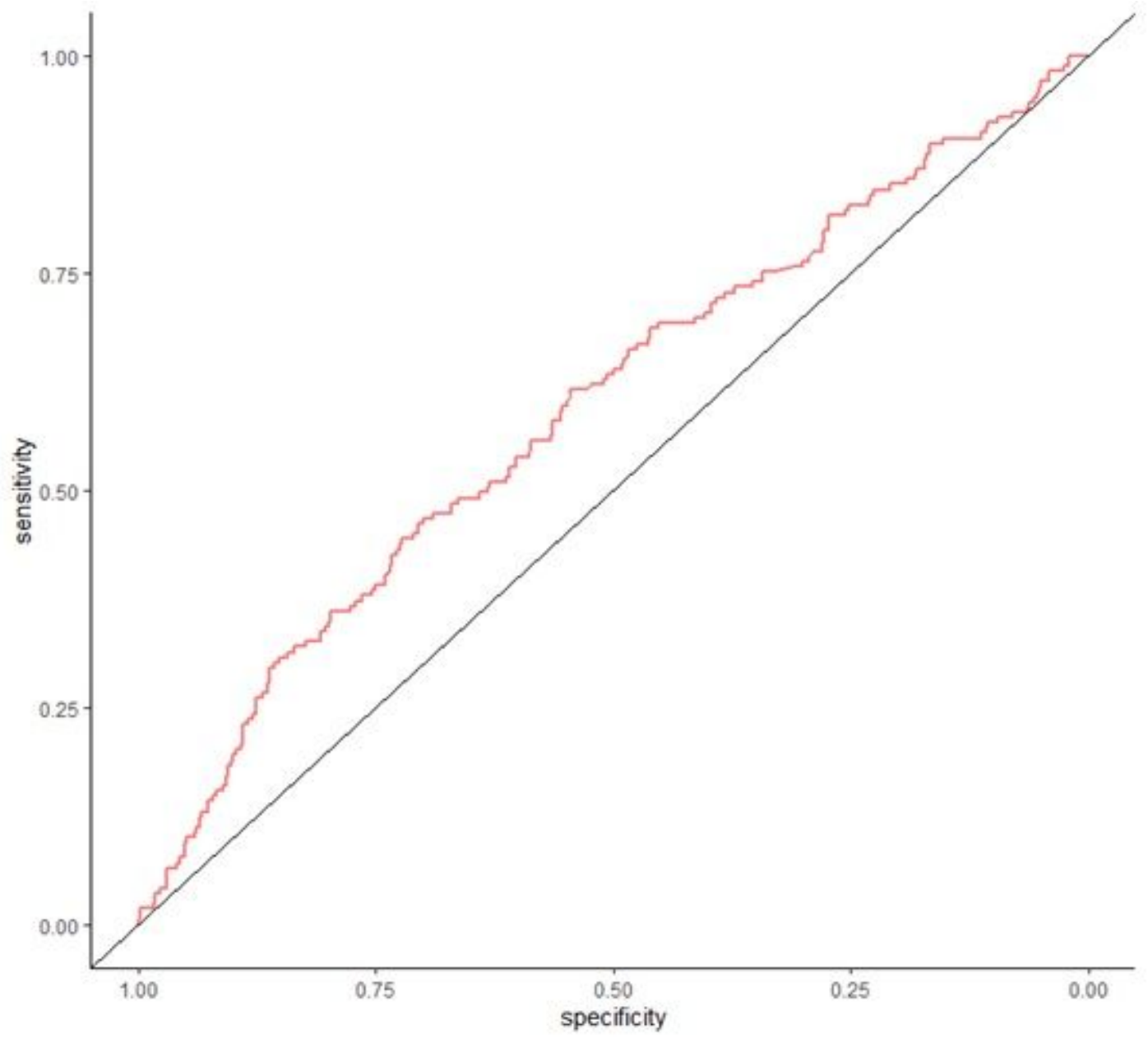

Figure 1

ROC curve of GGT/ALT in predicting vascular invasion for HBV-related HCC patients 
Strata $+{ }^{\prime}$ GGT/ALT =high + " GGT/ALT =low

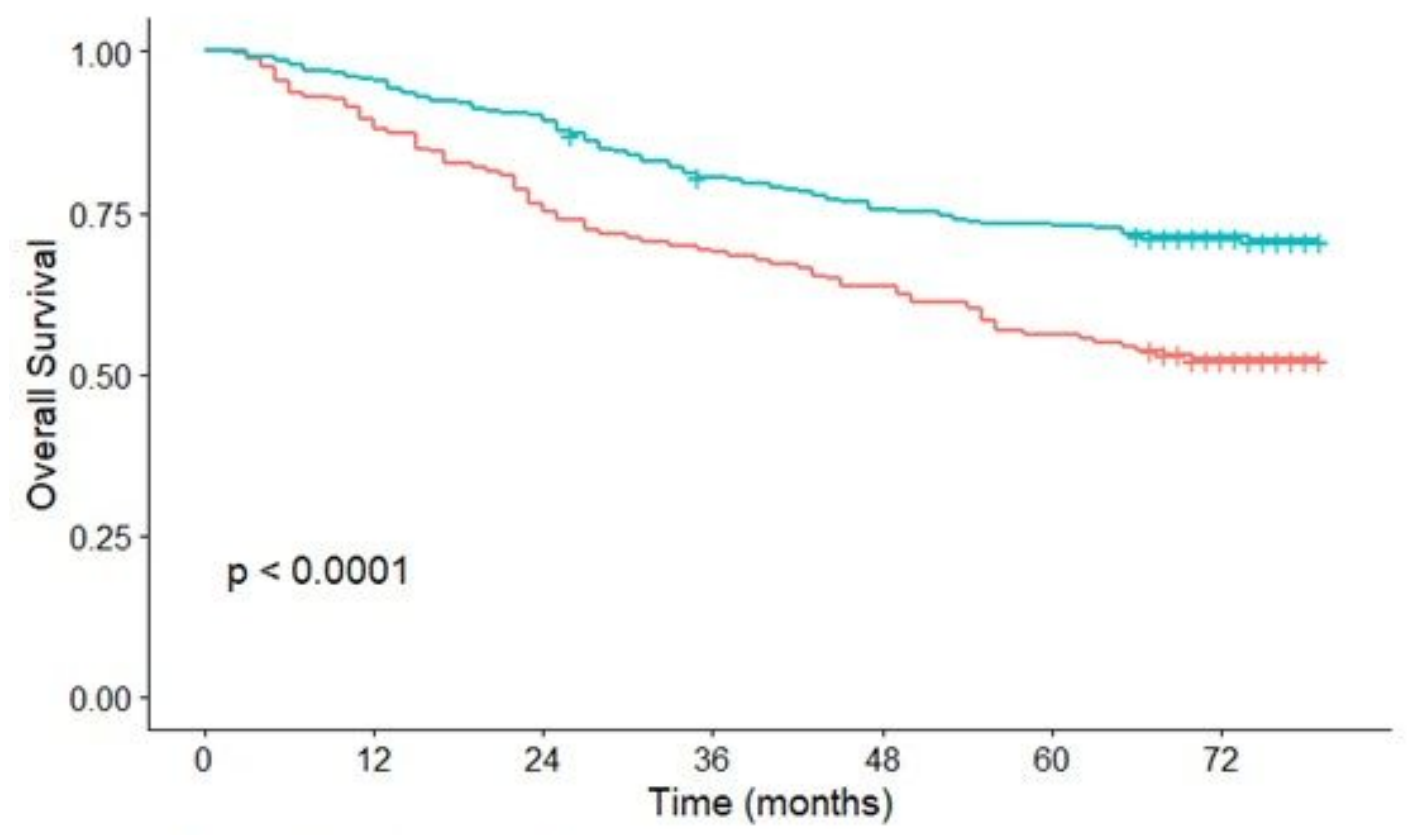

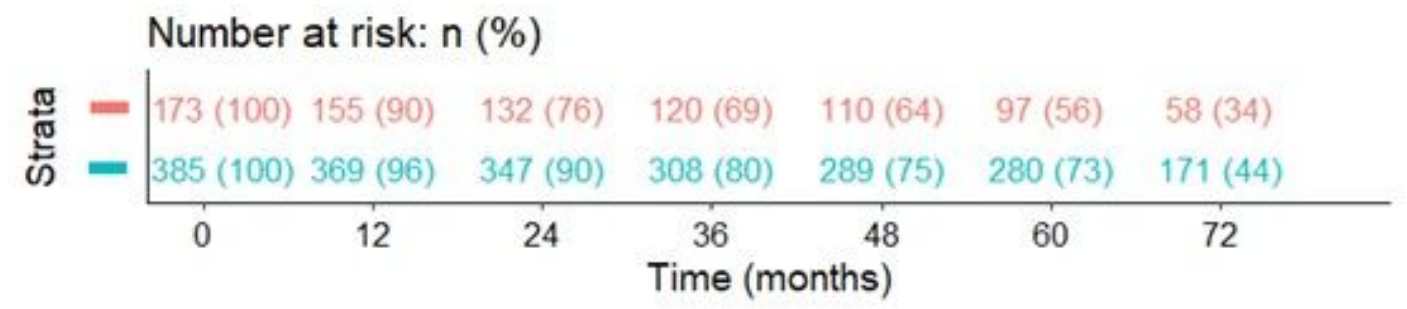

\section{Figure 2}

The analysis indicated that the high GGT/ALT resulted in poor overall survival rates. 

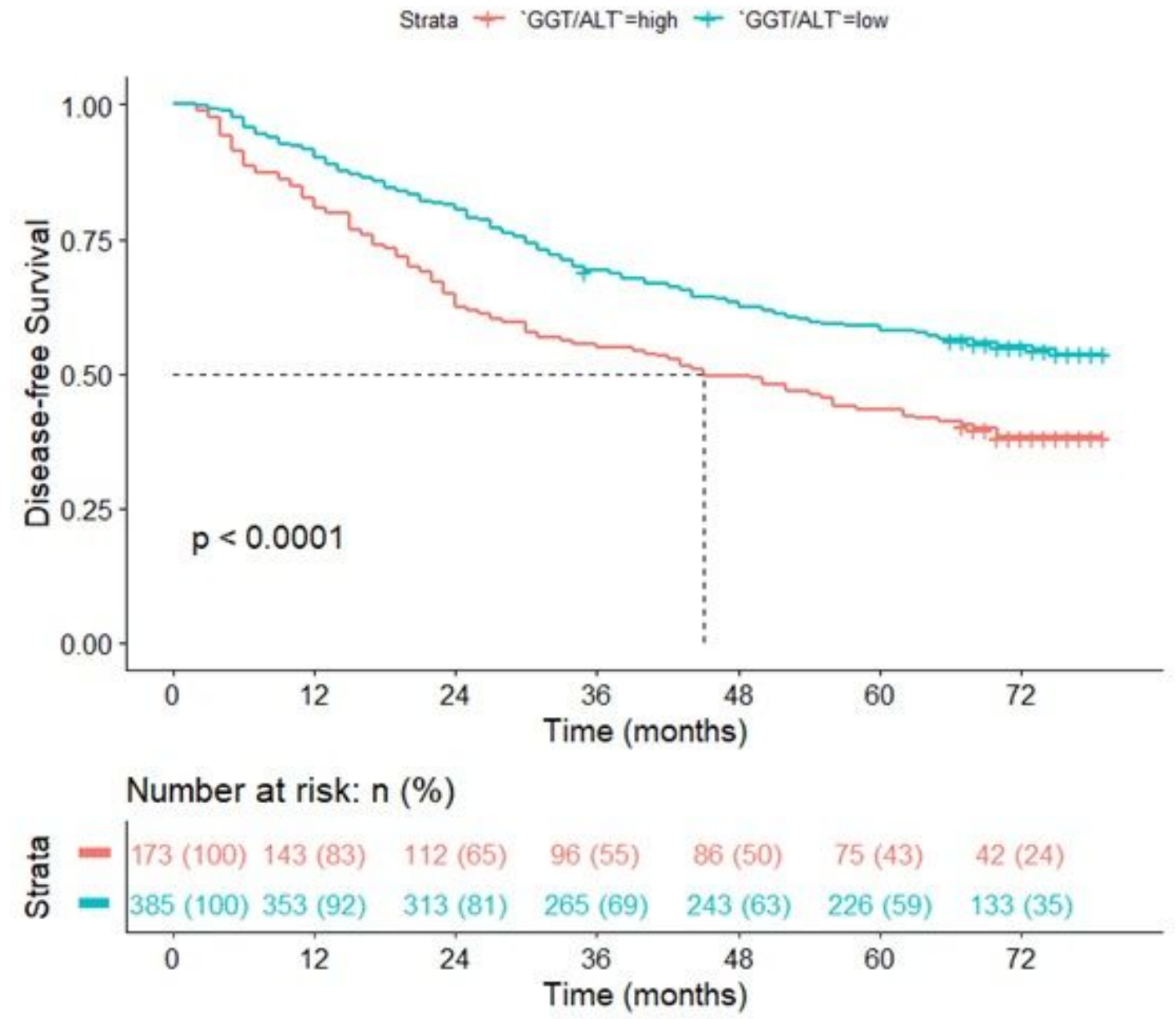

\section{Figure 3}

The analysis indicated that the high GGT/ALT resulted in poor disease-free survival rates. 